\title{
Uso de Plantas Medicinais por Gestantes em uma unidade Básica de Saúde de Juazeiro do Norte - CE
}

\author{
Use of Medicinal Plants by Pregnant women in a Bacic Health Unit of Juazeiro do Norte - CE \\ Uso de Plantas Medicinales por mujeres embarazadas em uma Unidad Básica de Salud de Juazeiro \\ do Norte - CE
}

Recebido: 22/02/2021 | Revisado: 01/03/2021 | Aceito: 15/03/2021 | Publicado: 22/03/2021

\author{
Rejane Cristina Fiorelli de Mendonça \\ ORCID: https://orcid.org/0000-0003-3729-1158 \\ Centro Universitário Dr. Leão Sampaio, Brasil \\ E-mail: rejanefiorelli@ leaosampaio.edu.br \\ Cícero Deivid Bezerra de Morais \\ ORCID: https://orcid.org/0000-0002-0192-5035 \\ Centro Universitário Doutor Leão Sampaio, Brasil \\ E-mail: deividbuarck@ hotmail.com \\ Francisco Leonardo da Silva Feitosa \\ ORCID: https://orcid.org/0000-0002-1072-4796 \\ Centro Universitário Dr. Leão Sampaio, Brasil \\ E-mail: flsfeitosa@gmail.com \\ José Leonardo Gomes Coelho \\ ORCID: https://orcid.org/0000-0001-6028-0807 \\ Universidade Leonardo da Vinci, Brasil \\ E-mail: leonardo-coelho-10@ hotmail.com \\ Francisca Stefane do Nascimento Andrade \\ ORCID: https://orcid.org/0000-0002-0847-8864 \\ Centro Universitário Dr. Leão Sampaio. Brasil \\ E-mail: stefanyandrade03@gmail.com \\ Linaria Martins Ferreira \\ ORCID: https://orcid.org/0000-0001-9355-6339 \\ Centro Universitário Dr. Leão Sampaio, Brasil \\ E-mail: linara_martins@hotmail.com \\ Lorena Monte Sousa \\ ORCID: https://orcid.org/0000-0002-2827-5845 \\ Centro Universitário Dr. Leão Sampaio, Brasil \\ E-mail: lorenamonte23@gmail.com \\ Isadora Gislene Lopes de Souza \\ ORCID: https://orcid.org/0000-0003-3076-7344 \\ Centro Universitário Dr. Leão Sampaio, Brasil \\ E-mail: isadoralsouza@hotmail.com \\ Paulo Jefter Marciel Maia \\ ORCID: https://orcid.org/0000-0003-4473-3938 \\ Centro Universitário Dr. Leão Sampaio, Brasil \\ E-mail: fisiojeftermaia@gmail.com \\ Karine Rocha da Cruz \\ ORCID: https://orcid.org/0000-0001-8591-0748 \\ Centro Universitário Dr. Leão Sampaio, Brasil \\ E-mail: kariineerocha@hotmail.com \\ Jaime Ribeiro Filho \\ ORCID: https://orcid.org/0000-0003-3126-6509 \\ Fundação Oswaldo Cruz, Brasil \\ E-mail: jaime.ribeiro@ fiocruz.br
}

\begin{abstract}
Resumo
Introdução: No período gestacional o corpo materno sofre inúmeras alterações fisiológicas em um curto espaço de tempo. $\mathrm{O}$ uso de produtos farmacoterapêuticos na gestação deve ser cauteloso já que muitas substâncias encontradas nesses produtos podem trazer riscos gravíssimos para a gestante. Objetivo: Avaliar o uso de plantas medicinais por gestantes atendidas em uma unidade básica de saúde de Juazeiro do Norte-CE. Método: O presente estudo é caracterizado como transversal e observacional de caráter quantitativo. Realizado com 15 gestantes de uma UBS de juazeiro do norte no período de outubro de 2019 a novembro de 2019. Resultados: No presente estudo foi avaliado o uso de produtos farmacoterapêuticos por gestantes atendidas em uma Unidade Básica de Saúde de Juazeiro do Norte, de início caracterizou-se o perfil socioeconômico, em seguida o histórico gestacional, e por fim verificou-se o uso de
\end{abstract}


plantas medicinais pelas gestantes, onde notou-se que $20 \%$ das gestantes utilizavam algum tipo de planta medicinal. Conclusão: Foi possível observar as plantas medicinais utilizadas pelas gestantes em uma Unidade Básica de Saúde de juazeiro do Norte-CE, podendo verificar que mesmo sendo realizada pela minoria das gestantes a prática de uso de produtos farmacoterapêuticos continua em alta atualmente. Desse modo, é de extrema importância que as mulheres em estado gestacional tenham o devido cuidado com a sua saúde quando se trata do uso de plantas medicinais, pois muitas das plantas medicinais não possuem estudos que indiquem sua eficácia e segurança, assim como os seus efeitos tóxicos.

Palavras-chave: Gestação; Plantas medicinais; Medicamentos.

\begin{abstract}
Introduction: In the gestational period, the maternal body undergoes numerous physiological changes in a short period of time. The use of pharmacotherapeutic products during pregnancy should be cautious since many substances found in these products can bring very serious risks to the pregnant woman. Objective: To evaluate the use of medicinal plants by pregnant women attended at a basic health unit in Juazeiro do Norte-CE. Method: The present study is characterized as cross-sectional and observational with a quantitative character. Conducted with 15 pregnant women from a UBS in Juazeiro do Norte from October 2019 to November 2019. Results: In the present study, the use of pharmacotherapeutic products by pregnant women attended at a Basic Health Unit in Juazeiro do Norte, was evaluated. the socioeconomic profile was characterized, followed by the gestational history, and finally, the use of medicinal plants by pregnant women was verified, where it was noted that $20 \%$ of pregnant women used some type of medicinal plant. Conclusion: It was possible to observe the medicinal plants used by pregnant women in a Basic Health Unit in juazeiro do Norte-CE, and it can be seen that even though it is performed by a minority of pregnant women, the practice of using pharmacotherapeutic products remains on the rise today. Thus, it is extremely important that women in gestational status take due care of their health when it comes to the use of medicinal plants, as many medicinal plants do not have studies that indicate their efficacy and safety, as well as their effects toxic.
\end{abstract}

Keywords: Gestation; Medicinal plants; Medicines.

\title{
Resumen
}

Introducción: En el período gestacional, el cuerpo materno sufre numerosos cambios fisiológicos en un corto período de tiempo. El uso de productos farmacoterapéuticos durante el embarazo debe ser cauteloso ya que muchas sustancias que se encuentran en estos productos pueden traer riesgos muy serios para la mujer embarazada. Objetivo: Evaluar el uso de plantas medicinales por parte de gestantes atendidas en una unidad básica de salud en Juazeiro do Norte-CE. Método: El presente estudio se caracteriza por ser de corte transversal y observacional con carácter cuantitativo. Realizado con 15 gestantes de una UBS de Juazeiro do Norte de octubre de 2019 a noviembre de 2019. Resultados: En el presente estudio se evaluó el uso de productos farmacoterapéuticos por parte de gestantes atendidas en una Unidad Básica de Salud de Juazeiro do Norte, iniciando el análisis socioeconómico. se caracterizó el perfil, seguido de la historia gestacional, y finalmente se verificó el uso de plantas medicinales por parte de gestantes, donde se constató que el 20\% de las gestantes usaba algún tipo de planta medicinal. Conclusión: se pudo observar las plantas medicinales utilizadas por gestantes en una Unidad Básica de Salud en juazeiro do Norte-CE, y se puede observar que si bien la práctica de uso de productos farmacoterapéuticos aún es alta, la práctica de uso de productos farmacoterapéuticos sigue siendo alto hoy. Por ello, es de suma importancia que las mujeres en estado gestacional cuiden debidamente su salud cuando se trata del uso de plantas medicinales, ya que muchas plantas medicinales no cuentan con estudios que indiquen su eficacia y seguridad, así como sus efectos tóxicos.

Palabras clave: Gestación; Plantas medicinales; Medicamentos.

\section{Introdução}

No período gestacional o corpo materno sofre inúmeras alterações fisiológicas em um curto espaço de tempo. Essas alterações ocorrem através de uma condição fisiológica complexa, sendo iniciadas desde o momento da nidação e estendendose por todo período gestacional até o término da lactação. E devido ao aparecimento de alguns efeitos indesejáveis durante o processo de gestação pode-se prática do uso de alguns tipos de produtos com ação medicamentosa como o uso de chás de plantas naturais para amenizar estes efeitos indesejáveis. E desta forma, pode-se a vir desenvolver reações tóxicas e afetem tanto a mãe como o feto (Costa, et al., 2010).

O uso de plantas medicinais com ação medicamentosa na gestação deve ser cauteloso já que muitas substâncias encontradas nesses produtos (especialmente os princípios ativos) podem trazer riscos para a gestante e principalmente para o feto, podendo estimular a mortalidade uterina e provocar aborto. Segundo Organização Mundial da Saúde (OMS), 80\% da população mundial não possui atendimento primário, o que acarreta o aumento na demanda do consumo plantas medicinais, os 
quais representam, muitas vezes, o único recurso terapêutico de muitas comunidades e grupos étnicos. Além disso, o Brasil contribui bastante para a larga utilização de fitoterápicos por possuir uma enorme biodiversidade e cultura no uso popular de plantas medicinais (Pontes, et al.,2012).

A utilização de determinadas plantas medicinais pode acarretar a embriotoxidade, que se refere a uma perturbação no desenvolvimento do embrião, independente da dose usada pela mãe. Essa toxidade é facilitada pela presença da circulação fetal, movimento de comunicação do feto ao meio externo do organismo materno ligados pela placenta que traz o dever de nutrir o feto. Por isso, é importante saber que assim como as ingestões de nutrientes trará benefícios ao desenvolvimento fetal, o uso de certas plantas medicinais pode gerar consequências irreversíveis a vida fetal podendo provocar até mesmo a morte do mesmo (Rodrigues, et al.,2011)

Produtos naturais com finalidade terapêutica é uma prática crescente no Brasil. Embora preparações obtidas de plantas medicinais sejam alternativas eficientes no tratamento de diversas doenças, uso irracional destes produtos pode contribuir para o desenvolvimento de distúrbios hepáticos, gástricos, teratogênicos e muitos outros danos à saúde dos usuários. Neste contexto, as gestantes constituem um grupo que merece atenção especial quanto ao monitoramento na utilização de produtos naturais, devido ao risco da exposição a substâncias químicas que podem comprometer a gestação e causar toxicidade para o embrião e/ou feto (Ribeiro, et al., 2013)

Partindo desse pressuposto, a presente pesquisa surge dos seguintes questionamentos: Qual o uso de plantas medicinais com ação medicamentosa na gestação?

Portanto, este estudo tem como objetivo avaliar o uso de plantas medicinais por gestantes atendidas em uma unidade básica de saúde de Juazeiro do Norte-CE. Sendo os objetivos específicos: traçar o perfil sociodemográfico das gestantes, relatar o histórico obstétrico das gestantes, descrever a percepção das gestantes quantos ao uso das plantas medicinais e verificar quais plantas medicinais são utilizadas pelas gestantes.

\section{Metodologia}

O presente estudo trata-se de um estudo transversal e observacional de caráter quantitativo. Os estudos observacionais objetivam-se em analisar se existe combinação entre um determinado fator e um desfecho sem intervir diretamente na relação verificada. O estudo transversal é um tipo de estudo onde consegue-se estimar a frequência com que um determinado evento de saúde se manifesta em um determinado grupo específico, além de outros fatores que estão ligados com ele. Para uma boa condução de um estudo transversal deve-se seguir as seguintes observações: Primeiramente definir o grupo de interesse, em seguida estudar essa determinada população utilizando a sua amostragem e por fim identificar a presença ou ausência de desfecho e exposição dos grupos estudados (Sucigan, et al.,2002; Bastos \& Duquia,2013)

A população do estudo foi constituída por 15 gestantes atendidas na Unidade Básica de Saúde no Bairro Vila Real Estratégia Saúde da Família (ESF 47) no município de Juazeiro do Norte, no período de outubro de 2019 a dezembro de 2019.

Foram incluídas no presente estudo gestantes maiores de dezoito anos, cadastradas na UBS do município de Juazeiro do Norte, em qualquer fase gestacional, podendo ser primíparas ou multíparas, com viabilidade de parto normal ou cesariano e que não fazem uso de medicamentos contínuos. Foram excluídas do estudo gestantes com síndromes hipertensivas crônicas ou gestacional, diabetes tipo I, tipo II ou diabetes gestacional, bem como grupos de riscos que utilizem cotidianamente medicamentos prescritos e que não aceitaram participar de forma livre e esclarecida da pesquisa.

A coleta foi iniciada pela fase de preparação constituída pela composição do levantamento literário, para construção de argumentações reflexivas e críticas embasadas na prática baseada em evidências, levantamento dos instrumentos de coleta de dados que será utilizado para a pesquisa, determinação da amostra e sequentemente a submissão ao comitê de ética e pesquisa do Centro Universitário Dr. Leão Sampaio - UNILEÃO. 
Após a aprovação do comitê de ética e pesquisa de Parecer $\mathrm{N}^{\circ} 3.778 .400$, iniciou-se o processo de informação e abordagem das gestantes quanto ao objetivos do estudo, esclarecimentos dos riscos e benefícios e após assinarem o termo de consentimento livre e esclarecido aplicou-se um questionário semiestruturado pelos próprios pesquisadores, o questionário foi dividido em três momentos, primeiro; a investigação do perfil socioeconômico das gestantes, segundo; a identificação do histórico obstétrico das gestantes e terceiro; a abordagem da investigação do nível de conhecimento de uso de plantas medicinais, que contemplassem o que elas sabem sobre o uso de plantas medicinais consumidas na gestação, quais plantas elas consomem e se conhecem os efeitos destas plantas medicinais para seu organismo. O questionário foi aplicado na Unidade Básica de Saúde do Bairro Vila real, ESF 47. O processo de coleta através do questionário foi realizado na UBS quando as gestantes aguardavam a consulta de pré-natal durante todas as segundas e quartas feiras do mês de novembro e dezembro.

Os dados coletados foram analisados seguidas das informações obtidas nos questionários e logo após tabelados e representados em gráficos do software Microsoft Office Excel 2010.

\section{Resultados e Discussão}

No presente estudo avaliou-se o uso de plantas medicinais em 15 gestantes atendidas em uma Unidade Básica de Saúde de Juazeiro do Norte-CE, tendo em vista nesta Unidade Básica de Saúde eram atendidas em médica de 20 gestantes.

A Tabela 1 aponta a caracterização do perfil socioeconômico, observou-se que 11 (onze) gestantes (73,3\%) moravam com uma a três pessoas, sendo que 10 (dez) gestantes $(66,6 \%)$ moram em casas alugadas, também foi possível observar que tanto as gestantes como seus pais possuíam baixa escolaridade. 7 (sete) gestantes (46,6\%) apresentaram renda familiar inferior a 1 salário mínimo, e 9 (nove) gestantes (60\%) não apresentam uma renda mensal fixa.

Tabela 1. Perfil Socioeconômico das gestantes.

Parametro socioeconomico

\begin{tabular}{l|l|l}
\hline Número de moradores & Uma a três pessoas & $11(73,3 \%)$ \\
\hline Tipo de Moradia & Alugada & $10(66,6 \%)$ \\
\hline Escolaridade do pai & Não sabem & $7(46,6 \%)$ \\
\hline Escolaridade da mãe & Até a $4^{\circ}$ série & $8(53,3)$ \\
\hline Escolaridade da gestante & Da $5^{\circ}$ a $8^{\circ}$ série & $7(46,6 \%)$ \\
\hline Renda familiar & Até 1 salário mínimo & $7(46,6)$ \\
\hline Renda da gestante & Nenhuma renda & $9(60 \%)$ \\
\hline Zona de moradia & Zona urbana & $10(66,6 \%)$ \\
\hline
\end{tabular}

Fonte: Morais e Mendonça (2019).

De acordo com o histórico gestacional das mesmas, destaca-se que a idade gestacional das participantes tem em média de 27,8 semanas de gestação, onde a idade gestacional menor foi de 22 semanas e a idade gestacional máxima foi de 35 semanas, foi observado que 6 (seis) gestantes (40\%) eram multíparas e se encontravam na segunda gestação e 11 (onze) gestantes $(73,3 \%)$ afirmam não ter sofrido nenhum tipo de aborto. Pode-se verificar que todas as gestantes (100\%) dessa determinada UBS realizavam o pré-natal de forma regular. 
Tabela 2. Histórico Gestacional.

\begin{tabular}{l|l|l}
\hline Parametro gestacional & Perfil prevalente & Representividade \\
\hline Número de gestações & 2 Gestações & $6(40 \%)$ \\
\hline Número de abortos & Não sofreu aborto & $11(73,3 \%)$ \\
\hline Pré-natal & Realiza & $15(100 \%)$ \\
Semana Gestacional & 25 semanas & $3(20 \%)$ \\
\hline
\end{tabular}

Fonte: Morais e Mendonça (2019).

Na Figura 1 verificou-se a percepção das gestantes sobre o uso de plantas medicinais na fase gestacional, no gráfico (A), aponta se as gestantes já utilizou plantas medicinais e observou-se que $97 \%$ das gestantes já utilizou plantas medicinais e $3 \%$ nunca utilizou plantas medicinais, foi analisado no gráfico (B) que (36\%) das gestantes afirmaram que o uso de plantas medicinais não fazem mal no período gestacional, $36 \%$ responderam que as plantas medicinais fazem menos mal que os medicamentos convencionais e $28 \%$ responderam que as plantas medicinais fazem tão mal quanto os medicamentos convencionais.

Partindo para o gráfico (C), observou-se que (7\%) das gestantes acham que as plantas medicinais podem ajudar a gravidez, $43 \%$ acham que podem atrapalhar a gravidez e (50\%) acham que o uso dessas plantas não interfere na gravidez. Conforme o gráfico (D), 20\% das gestantes usaram algum tipo de planta medicinal na gestação e (80\%) não utilizaram nenhum tipo de planta durante a gestação.

Figura 1. Percepção das gestantes sobre a utilização de plantas medicinais na fase gestacional.

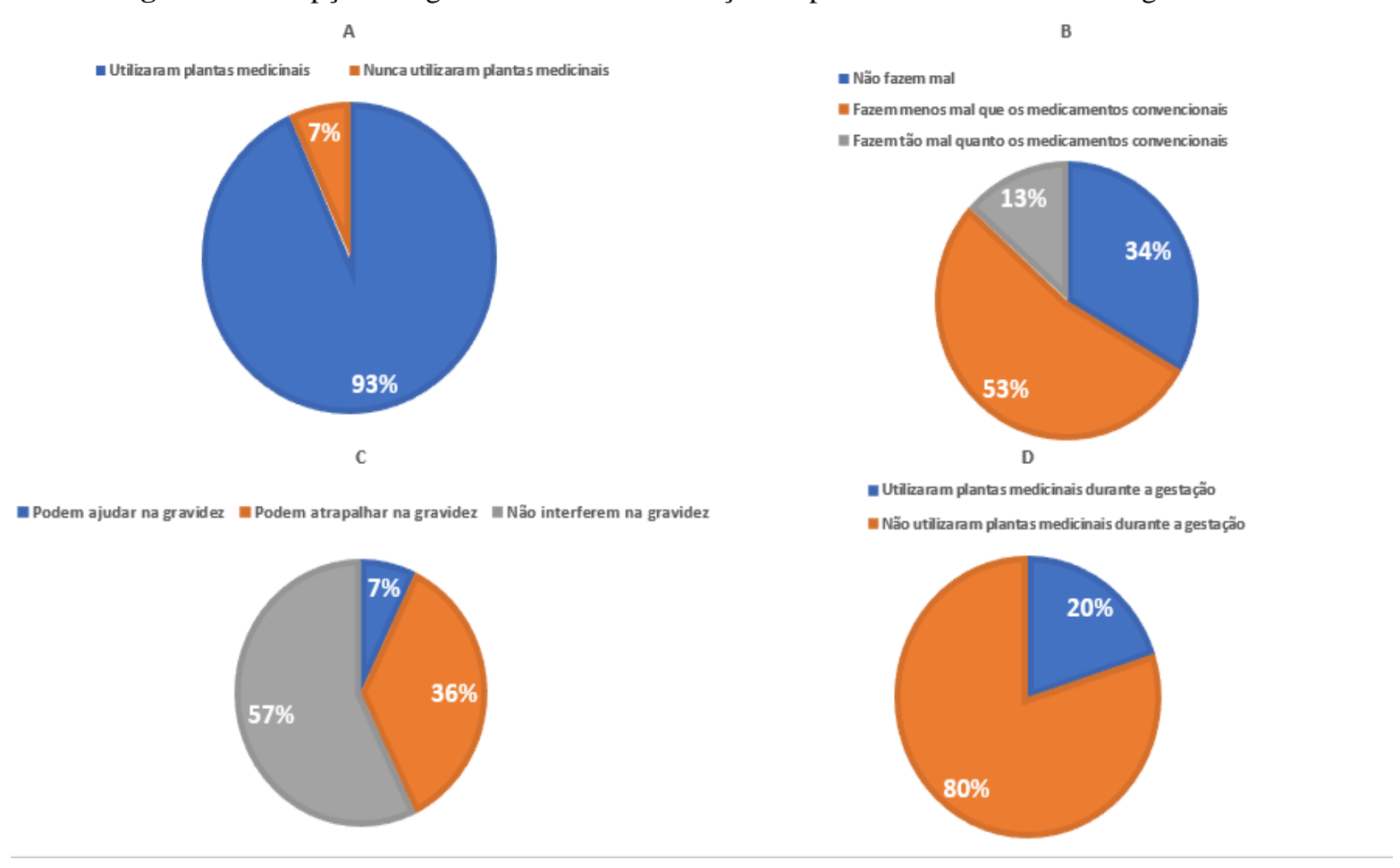

Fonte: Morais e Mendonça (2019).

Por fim, analisou-se o uso de quais tipos de plantas medicinais estas gestantes consumiram no período gestacional. Onde pode-se observar na Tabela 3 que as plantas medicinais citadas foram: Camomila (Matricaria camomita), Gengibre 
(Zingiber officinale), Laranja (Citrus sinensis) todas utilizadas em forma de chás. E quanto a medicamentos industrializados $100 \%$ não utilizaram, apenas suplementação com Sulfato Ferroso.

Tabela 3. Produtos Farmacoterapêuticos Utilizados Pelas Gestantes.

\begin{tabular}{l|l|} 
Produtos Farmacoterapêuticos & Forma de Utilização \\
\hline Camomila (Matricaria camomila) & Chá das Flores \\
\hline Gengibre (Zingiber officinale) & Chá da Raiz \\
\hline Laranja (Citrus sinesis) & Chá das folhas \\
\hline
\end{tabular}

Fonte: Morais e Mendonça (2019).

Essa pesquisa foi realizada com gestantes em uma Unidade Básica de Saúde de Juazeiro do norte ceara, onde observou-se a prática de uso de plantas medicinais na fase gestacional.

O uso de plantas medicinais com finalidade de tratamento de enfermidade é uma prática presenta nas diversas culturas há séculos, e que até hoje é realizada. Por serem de fácil acesso, baixo custo e pela crença que as plantas medicinais podem ser menos nocivas e mais eficazes, faz com que essa prática permaneça atualmente. Outra questão é a automedicação, prática de ingerir substâncias sem qualquer prescrição médica, isso se dá pela falta de acesso ao atendimento médico, devido ao alto custo de planos de saúde ou até mesmo a precariedade de serviços públicos de saúde (Abreu da Sailva., et al, 2018)

Um estudo realizado por Bitu e colaboradores (2015) em mercados de Juazeiro do Norte-CE revelou que o uso e a comercialização nessa região é uma prática comum com fins terapêuticos. Na entrevista procurou-se saber a quantidade de plantas utilizadas, a percepção das gestantes sobre o ouso das mesmas, tentando relacionar como o meio socioeconômico, seu histórico gestacional, sua prática e percepção pode interferir na gestação. Na figura 1 pode-se observar que $20 \%$ das gestantes usaram algum tipo de planta medicinal.

Segundo a Figura 1 a prevalência de uso de plantas medicinais na gestação está totalmente relacionada com a população de uma classe social menor, ou seja, de baixa renda, baixa escolaridade e baixo acesso a informações.

No estudo foram coletados o uso de 3 tipos de plantas: A folha da laranja (Citrus sinesis), a camomila (Matricaria camomila) e o gengibre (Zingiber officinale), podendo perceber que na UBS pesquisada o uso de plantas medicinais é realizado pela minoria das gestantes.

Estudos prévios demostraram os riscos dessas determinadas plantas sobre a saúde da mãe e do feto. Morais e colaboradores (2012) mostraram que o uso da camomila (Matricaria camomila) possui efeito abortivo. Amorim e colaboradores (2013) mostraram que o Gengibre (Zingiber officinale) apesar de trazer alguns benefícios para a gestante como a redução de náuseas e vômitos, também apresenta riscos como: efeito abortivo, parto prematuro, anomalias congênitas, placenta previa e pré-eclâmpsia. Em uma consulta na literatura não foi possível encontrar a respeito das contra-indicações sobre o uso da laranja (Citrus sinesis).

No geral pode-se afirmar que embora neste estudo tenha se mostrado um percentual pequeno em relação ao uso de plantas medicinais é importante ressaltar que se faz necessário adotar medidas que mostrem os riscos e benefícios da utilização dessas plantas, para que as gestantes possam consumi-las de forma segura. Algumas plantas possuem na sua composição metabólitos secundários como: os alcaloides, antraquinonas, flavonoides, cumarinas e terpenos, os quais são relacionados à possíveis danos à saúde da gestante e do bebê. Desse modo, é de extrema importância que as mulheres em estado gestacional tenham o devido cuidado com a sua saúde quando se trata do uso de plantas medicinais, pois muitas das plantas medicinais não possuem estudos que indiquem sua eficácia e segurança, assim como os seus efeitos tóxicos. (Abreu da Silva, et al,2018). 


\section{Conclusão}

Foi possível observar as plantas medicinais, camomila; gengibre e laranja utilizados pelas gestantes em uma Unidade Básica de Saúde de juazeiro do norte cearam, podendo verificar que mesmo sendo realizada pela minoria das gestantes a prática de uso de produtos farmacoterapêuticos continua em alta atualmente.

Desse modo, é de extrema importância que as mulheres em estado gestacional tenham o devido cuidado com a sua saúde quando se trata do uso de plantas medicinais, pois muitas das plantas medicinais não possuem estudos que indiquem sua eficácia e segurança, assim como os seus efeitos tóxicos, o que pode ocasionar problemas para a mãe e para o feto.

\section{Referências}

Amorim, A., Ferreira, A. R. R., \& Carrapiço, E. (2013). Ginger for the treatment of nausea and vomiting of pregnancy: evidence-based review Gengibre no tratamento da náusea e vómito da gravidez: revisão baseada na Evidência. Acta Obstet Ginecol Port, 7(2), 103-108.

Angueira, A. R., Ludvik, A. E., Reddy, T. E., Wicksteed, B., Lowe, W. L., \& Layden, B. T. (2015). New insights into gestational glucose metabolism: lessons learned from 21st century approaches. Diabetes, 64(2), 327-334.

Bastos, J. L. D., \& Duquia, R. P. (2007). Um dos delineamentos mais empregados em epidemiologia: estudo transversal. Scientia Medica, 17(4), 229-232.

Bitu, V. D. C. N., Bitu, V. D. C. N., Matias, E. F. F., de Lima, W. P., da Costa Portelo, A., Coutinho, H. D. M., \& de Menezes, I. R. A. (2015). Ethnopharmacological study of plants sold for therapeutic purposes in public markets in Northeast Brazil. Journal of Ethnopharmacology, 172, $265-272$.

Borges, V. M., Moura, F., Cerdeira, C. D., \& Barros, G. B. S. (2018). Uso de medicamentos entre gestantes de um município no suL de Minas Gerais, Brasil. Infarma-Ciências Farmacêuticas, 30(1), 30-43.

Brum, L. F. D. S., Pereira, P., Felicetti, L. L., \& Silveira, R. D. D. (2011). Utilização de medicamentos por gestantes usuárias do Sistema Único de Saúde no município de Santa Rosa (RS, Brasil). Ciência \& Saúde Coletiva, 16, 2435-2442.

dos Santos Souza, J. S., Gomes, E. C., Rocha, T. C., \& Böger, B. (2017). Uso de plantas medicinais por comunidades do município de Curitiba.Divers@!,10(2),91-97.

Mazaki-Tovi, S., Kanety, H., Pariente, C., Hemi, R., Yissachar, E., Schiff, E., \& Sivan, E. (2011). Insulin sensitivity in late gestation and early postpartum period: the role of circulating maternal adipokines. Gynecological Endocrinology, 27(9), 725-731.

Melo, S. C. C. S. D., Pelloso, S. M., Carvalho, M. D. D. B., \& Oliveira, N. L. B. D. (2009). Uso de medicamentos por gestantes usuárias do Sistema Único de Saúde. Acta Paulista de Enfermagem, 22(1), 66-70.

Morais, R. K. A., de Souza, J. F., do Monte, N. L., de Andrade, E. T. S., \& de Araújo, C. R. F. Efeitos Teratogênicos E Abortivos De Plantas Medicinais E Fitoterápicos: Gestantes Do Serviço Público De Saúde Em Um Relato De Experiência.

Nomura, R. M. Y., Miyadahira, S., \& Zugaib, M. (2009). Avaliação da vitalidade fetal anteparto. Rev Bras Ginecol Obstet, 31 (10), $513-26$.

Pontes, S. M. (2012). Utilização de plantas medicinais potencialmente nocivas durante a gestação. Comunicação em ciêNcias da Saúde, $23(4), 305-311$.

Rodrigues, H. G., Meireles, C. G., Lima, J. T. S., Toledo, G. P., Cardoso, J. L., \& Gomes, S. L. (2011). Efeito embriotóxico, teratogênico e abortivo de plantas medicinais. Revista brasileira de plantas medicinais, 13(3), 359-366.

Santana, L. L., \& Da Silva, A. C. A. (2019). Os Riscos do Uso de Plantas Medicinais Durante o Período Gestacional. Acta Toxicológica Argentina, 26(3).

Costa, E. S., Pinon, G. M. B., Costa, T. S., de Araújo Santos, R. C., Nóbrega, A. R., \& de Sousa, L. B. (2010). Alterações fisiológicas na percepção de mulheres durante a gestação. Revista da Rede de Enfermagem do Nordeste, 11(2), 86-93.

Soma-Pillay, P., Catherine, N. P., Tolppanen, H., Mebazaa, A., Tolppanen, H., \& Mebazaa, A. (2016). Physiological changes in pregnancy. Cardiovascular journal of Africa, 27(2), 89.

Tkachenko, O., Shchekochikhin, D., \& Schrier, R. W. (2014). Hormones and hemodynamics in pregnancy. International journal of endocrinology and metabolism, 12(2). 